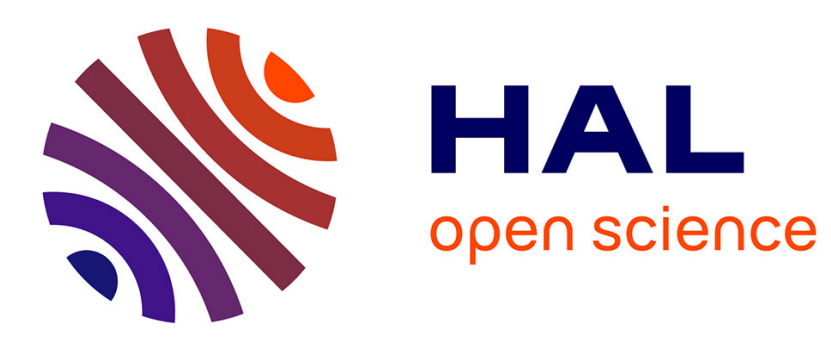

\title{
Aerodynamic force and Lamb vector field in compressible unsteady flows
}

Mario Ostieri, Renato Tognaccini, Didier Bailly, Daniel Destarac

\section{To cite this version:}

Mario Ostieri, Renato Tognaccini, Didier Bailly, Daniel Destarac. Aerodynamic force and Lamb vector field in compressible unsteady flows. AIAA Aerospace Sciences Meeting, 2018, Jan 2018, KISSIMMEE, United States. 10.2514/6.2018-0548 . hal-02475924

\section{HAL Id: hal-02475924 https://hal.science/hal-02475924}

Submitted on 12 Feb 2020

HAL is a multi-disciplinary open access archive for the deposit and dissemination of scientific research documents, whether they are published or not. The documents may come from teaching and research institutions in France or abroad, or from public or private research centers.
L'archive ouverte pluridisciplinaire HAL, est destinée au dépôt et à la diffusion de documents scientifiques de niveau recherche, publiés ou non, émanant des établissements d'enseignement et de recherche français ou étrangers, des laboratoires publics ou privés. 


\title{
Aerodynamic force and Lamb vector field in compressible unsteady flows
}

\author{
Mario Ostieri* ${ }^{\dagger}$, - Renato Tognaccini ${ }^{\ddagger}$ \\ Università di Napoli Federico II, Napoli, 80125, Italia \\ Didier Bailly ${ }^{\S}$, Daniel Destarac ${ }^{\mathbb{I}}$ \\ ONERA-The French Aerospace Lab, F92190 Meudon, France
}

\begin{abstract}
A vorticity based theory for the computation and analysis of the aerodynamic force in steady and unsteady compressible flows is presented. It is the extension of a recent decomposition developed for incompressible flows. The breakdown in reversible and irreversible contributions is presented and a comparison with an unsteady thermodynamic drag breakdown is discussed. In particular, two physically completely different flows are analyzed: a pitching airfoil in subsonic flows and an airfoil natural buffet. A sensitivity analysis of the vortical method on the total force computation is presented. Then the breakdown results are compared and discussed.

*Ph.D. student, Dipartimento di Ingegneria Industriale, mariostieri@gmail.com

†Associate professor, Dipartimento di Ingegneria Industriale, renato.tognaccini@unina.it, +39 0817682179, AIAA senior member.

${ }^{\S}$ Research Engineer, Aerodynamics, Aeroelasticity and Acoustics Department, didier.bailly@onera.fr

ITResearch Engineer, Aerodynamics, Aeroelasticity and Acoustics Department, daniel.destarac@onera.fr
\end{abstract}

Corresponding author. 


\section{Nomenclature}

\begin{tabular}{|c|c|}
\hline$a$ & $=\quad$ speed of sound \\
\hline $2 b$ & $=$ plate length \\
\hline$c$ & $=$ airfoil chord \\
\hline$C_{l}$ & $=$ lift coefficient \\
\hline$C_{l_{b}}$ & $=$ lift coefficient by $\boldsymbol{F}_{b}$ \\
\hline$C_{l_{\text {rev }}}$ & $=$ reversible lift coefficient \\
\hline$C_{d}$ & $=$ drag coefficient \\
\hline$C_{d_{b}}$ & $=$ drag coefficient by $\boldsymbol{F}_{b}$ \\
\hline$C_{d_{i}}$ & $=$ lift-induced drag coefficient \\
\hline$C_{d_{i r r}}$ & $=$ irreversible drag coefficient \\
\hline$C_{d_{r e v}}$ & $=$ reversible drag coefficient \\
\hline$C_{d_{u i}}$ & $=$ unsteady lift-induced drag coefficient \\
\hline$C_{d_{v}}$ & $=$ viscous drag coefficient \\
\hline$C_{d_{w}}$ & $=$ wave drag coefficient \\
\hline$C_{d_{\ell}}$ & $=$ vortex force drag coefficient \\
\hline$D_{i}$ & $=$ lift-induced drag \\
\hline$D_{m}$ & $=$ motion drag \\
\hline$D_{p a}$ & $=$ acoustic propagation drag \\
\hline$D_{u i}$ & $=$ unsteady lift-induced drag \\
\hline$D_{v}$ & $=$ viscous drag \\
\hline$D_{w}$ & $=$ wave drag \\
\hline $\boldsymbol{F}$ & $=$ aerodynamic force \\
\hline $\boldsymbol{F}_{a}$ & $=$ Wu's full aerodynamic force expression \\
\hline $\boldsymbol{F}_{b}$ & $=$ Mele and Tognaccini full aerodynamic force expression \\
\hline $\boldsymbol{F}_{\text {irr }}$ & $=$ irreversible part of the aerodynamic force \\
\hline $\boldsymbol{F}_{\text {rev }}$ & $=$ reversible part of the aerodynamic force \\
\hline $\boldsymbol{F}_{t}$ & $=$ unsteady contribution to aerodynamic forces \\
\hline $\boldsymbol{F}_{t *}$ & $=$ modified $\boldsymbol{F}_{t}$ \\
\hline $\boldsymbol{F}_{t b}$ & $=$ contribution to aerodynamic force \\
\hline $\boldsymbol{F}_{\tau}$ & $=\boldsymbol{F}_{t}+\boldsymbol{F}_{S_{b}}$ \\
\hline$h$ & $=$ enthalpy \\
\hline$k$ & $\bar{\omega} c / 2 V \infty$ reduced frequency \\
\hline$H$ & $=$ total enthalpy \\
\hline$K_{p v}$ & $=$ gas heat capacity ratio \\
\hline I & $=$ hydrodynamic impulse \\
\hline$\ell$ & $=$ Lamb vector \\
\hline$m_{\rho}$ & $=$ compressible correction term \\
\hline$M_{\infty}$ & $=$ freestream Mach number \\
\hline $\boldsymbol{n}=\left[n_{x}, n_{y}, n_{z}\right]^{T}$ & $=$ unit normal vector \\
\hline$p$ & $=$ pressure \\
\hline$r$ & $=$ position vector \\
\hline$R$ & $=$ gas constant \\
\hline$R^{+}$ & $=u+2 /\left(K_{p v}-1\right)$ Riemmann invariant \\
\hline$R e_{\infty}$ & $=$ freestream Reynolds number \\
\hline$S_{f a r}, S_{b}$ & $=$ far field and body surfaces \\
\hline
\end{tabular}




$\begin{array}{ll}T & =\text { oscillation period } \\ u_{i r r} & =\text { irreversible velocity } \\ V=\left(V_{\infty}+u, v, w\right)^{T} & =\text { velocity vector } \\ V_{\infty} & =\text { freestream velocity } \\ x, y, z & =\text { cartesian coordinates } \\ x_{s}, y_{s} & =\text { max } x \text { and } y \text { values that define intgration domain } \\ x_{w} & =\text { wake station } \\ x_{w} & =\text { wake station vector } \\ \alpha, \bar{\alpha} & =\text { pitching law motion and amplitude } \\ \mu & =\text { dynamic viscosity } \\ \Delta_{\mu} & =\text { contribution to aerodynamic force } \\ \tau_{R} & =\text { Reynolds stress tensor } \\ \tau_{v} & =\text { viscous stress tensor } \\ \mathcal{V} & =\text { computational domain } \\ \mathcal{V}_{c} & =\text { complementary volume } \\ \mathcal{V}_{v} & =\text { viscous volume } \\ \mathcal{V}_{w} & =\text { shock wave volume } \\ \mathcal{V}_{w d} & =\text { shock wake volume } \\ \gamma & =\text { vorticity sheet distribution } \\ \bar{\omega} & =\text { angular frequency } \\ \omega & =\text { vorticity } \\ \rho & =\text { density } \\ \xi & =\text { integration variable }\end{array}$

\section{Introduction}

Unsteady flow phenomena are increasingly becoming important in the present industrial framework. Not only trendy applications like drones or MAVs, but also rotorcraft applications, noise reduction, or, more generally, physical insight into natural flight like birds or insects one, require accurate prediction of unsteady flow vortical structures, directly linked to force generation. During last decades, many theories have been developed to physically link aerodynamic force generation to local flow properties, like entropy or vorticity. Betz [1] introduced a so-called farfield method with which irreversible drag production could be computed by a wake survey of the flow, instead of stress analysis on the body. Oswatitsch [2] introduced the entropy drag concept, with which irreversible drag could be associated to local entropy production. Maskell [3] proposed a method to compute reversible drag effects, in particular the lift-induced drag, in incompressible flow, linking it to the vorticity on the Trefftz plane. Paparone and Tognaccini [4] and Van der Voreen and Destarac [5] proposed drag breakdown methods for steady flows, with which total drag could be separated in lift-induced, viscous and wave components, directly linking these components to local entropy production. Tognaccini also discussed the case of "powered" configurations [6]. These applications, however, were only limited to steady flows.

Gariepy et al. [7] proposed a first extension of Van der Vooren and Destarac method to unsteady flows. Toubin and Bailly [8] developed another extension of the steady breakdown theory, with application to complex two- and three-dimensional configurations. Finally Toubin et al. [9] further developed the method, introducing a drag component that accounts for acoustic propagation effects, with an application to a detached-eddy simulation of buffet phenomenon around an airfoil.

A new exact theory for the incompressible aerodynamic force computation was introduced by $\mathrm{Wu}$ et al [10]. It relies on the local Lamb vector field (the cross-product of vorticity and velocity), non negligible in a limited region of the flow only (mid field property). Marongiu and Tognaccini [11] proposed an extension to turbulent flows of this theory, with applications to steady and unsteady two-dimensional flows. Marongiu et al. [12] succeeded in giving a rigorous definition of reversible steady force effects (lift and lift-induced force components) in terms of vortex force.

A first extension of this theory to compressible flows was already proposed by Wu et al. [13], and further developed by Liu et al. [14],[15]. Mele and Tognaccini [16] retrieved the mid field property, proposing an alternative exact 
formula, with applications to two-dimensional transonic and supersonic flows. Mele et al. [17] successfully extended definition of lift-induced drag to compressible regime, adding to the vortex force a compressibility correction term. Finally, Ostieri et al. [18] applied this method to the analysis of a transonic aircraft configuration, and proposed a new definition of viscous and wave drag components.

Very recently, Ostieri et al. [19],[20] focused on the application of Wu's theory to unsteady flows. They introduced an alternative aerodynamic force expression, more accurate when dealing with moving rigid grid simulations, and introduced a new definition of dynamic force derivatives, thanks to a direct link with classical inviscid theories.

In present paper, an extension of Ostieri et al. [19],[20] theory to compressible flows is proposed. Numerical applications have been performend on a pitching airfoil in subsonic flow and an airfoil buffet test case, already proposed by Toubin and Bailly [8]. A sensitivity analysis of the Lamb-vector based theory to the integration domain is firstly presented for both test cases. The vortical decomposition then has been compared with drag breakdown computations performed by Toubin and Bailly [8], showing some analogies between reversible and irreversible effects found by these two methods.

\section{Aerodynamic drag breakdown and local irreversible velocity}

A brief summary of the thermodynamic drag breakdown theory [8, 9] is here reported. Considering a compressible unsteady viscous flow around a moving rigid body, the aerodynamic drag $D$ can be so defined:

$$
D=D_{v}+D_{w}+D_{u i}+D_{m}
$$

where

$$
\begin{gathered}
D_{v}=\int_{S_{v}}\left[-\rho\left(u_{i r r}-u_{\infty}\right) \boldsymbol{V}+\boldsymbol{\tau}_{v}\right] \cdot \boldsymbol{n} \mathrm{d} S-\int_{\mathcal{V}_{v}} \frac{\partial \rho\left(u-u_{\infty}\right)}{\partial t} \mathrm{~d} \mathcal{V} \\
D_{w}=\int_{S_{w}}\left[-\rho\left(u_{i r r}-u_{\infty}\right) \boldsymbol{V}+\boldsymbol{\tau}_{v}\right] \cdot \boldsymbol{n} \mathrm{d} S-\int_{\mathcal{V}_{w}} \frac{\partial \rho\left(u-u_{\infty}\right)}{\partial t} \mathrm{~d} \mathcal{V}+ \\
-\int_{\mathcal{V}_{w d}}\left[\frac{\partial \rho\left(u-u_{i r r}\right)}{\partial t}+\frac{1}{u_{i r r}} \frac{\partial p}{\partial t}\right] \mathrm{d} \mathcal{V} \\
D_{u i}=\int_{S_{\text {far }}}\left[-\rho\left(u-u_{i r r}\right) \boldsymbol{V}-\left(p-p_{\infty}\right) \boldsymbol{i}_{x}\right] \cdot \boldsymbol{n} \mathrm{d} S-\int_{\mathcal{V}_{c}}\left[\frac{\partial \rho\left(u-u_{i r r}\right)}{\partial t}+\frac{1}{u_{i r r}} \frac{\partial p}{\partial t}\right] \mathrm{d} \mathcal{V} \\
D_{m}=-\int_{S_{b}} \rho\left(u-u_{\infty}\right) \boldsymbol{V} \cdot \boldsymbol{n} \mathrm{d} S
\end{gathered}
$$

In these equations $\boldsymbol{V}=[u, v, w]^{T}$ is the velocity, $u_{\infty}$ is the freestream velocity, $\rho$ is the density, $p$ is the pressure, $\boldsymbol{\tau}_{v}$ is the viscous stress tensor, $\boldsymbol{n}$ is the normal versor, $\boldsymbol{i}_{x}$ is the $x$-axis versor, aligned with the free-stream velocity. $D_{u i}$ is the so-called unsteady-induced drag, $D_{v}$ is the viscous drag, $D_{w}$ is the wave drag and $D_{m}$ is a component that takes into account for the body motion, and is usually negligible. $u_{i r r}$ is the irreversible velocity [5],[8], so defined

$$
u_{i r r}=u_{\infty} \sqrt{1+\frac{2 \Delta H}{u_{\infty}^{2}}-\frac{2}{\left(K_{p v}-1\right) M_{\infty}^{2}}\left(e^{\left(\left(K_{p v}-1\right) \Delta s\right) / K_{p v} R}-1\right)}
$$

where $R$ is the gas constant, $K_{p v}$ is the heat capacity ratio of the gas, $H=h+0.5 V^{2}$ is the total enthalpy ( $h$ is the enthalpy) and $s$ is the entropy.

The viscous, wave and shock wave regions $\mathcal{V}_{v}, \mathcal{V}_{w}, \mathcal{V}_{w d}$ and their boundary surfaces can be selected by proper sensors [8] in the whole integration domain $\mathcal{V}$. The complementary domain $\mathcal{V}_{c}$ is defined as $\mathcal{V} \backslash\left(\mathcal{V}_{w} \cup \mathcal{V}_{w d} \cup \mathcal{V}_{v}\right)$.

Toubin et al. [9] also proposed a decomposition of the unsteady drag $D_{i}$ :

$$
\begin{gathered}
D_{i}=\int_{S_{f a r}}\left[-\rho\left(u-u_{i r r}\right) \boldsymbol{V}-\left(p-p_{\infty}\right) \boldsymbol{i}_{x}\right] \cdot \boldsymbol{n} \mathrm{d} S-\int_{\mathcal{V}_{c}}\left[\rho\left(u-u_{i r r}\right)\left(\frac{1}{a}-\frac{1}{u_{i r r}}\right) \frac{\partial u}{\partial t}\right] \mathrm{d} \mathcal{V} \\
D_{p a}=\int_{\mathcal{V}_{c}} \rho \frac{u-u_{i r r}}{a} \frac{\partial R^{+}}{\partial t} \mathrm{~d} \mathcal{V}
\end{gathered}
$$

where $a$ is the local speed of sound, $R^{+}=u+2 a /\left(K_{p v}-1\right)$ is the Riemann invariant. $D_{p a}$ is a drag component that takes into account for acoustic propagation effects, $D_{i}$ is what they call lift-induced drag, and $D_{u i}=D_{i}+D_{p a}$. 
The breakdown is based on thermodynamic assumptions to define the irreversible velocity. It is numerically robust since it doesn't rely on gradients computation. It can also take into account for spurious drag effects due to numerical discretization.

\section{Aerodynamic force and Lamb vector field}

\section{A. Incompressible regime}

Considering a viscous unsteady incompressible flow around a moving body, Wu et al. [10] derived an alternative exact expression of the aerodynamic force in terms of the vortex force $\boldsymbol{F}_{\ell}$ :

$$
\boldsymbol{F}_{a}=\boldsymbol{F}_{\ell}+\boldsymbol{F}_{t}+\boldsymbol{F}_{S_{f a r}}+\boldsymbol{F}_{S_{b}}+\boldsymbol{F}_{t_{b}}+\Delta_{\mu}
$$

where

$$
\begin{aligned}
\boldsymbol{F}_{\ell}=-\int_{\mathcal{V}} \rho \boldsymbol{\ell} \mathrm{d} \mathcal{V}, & \boldsymbol{F}_{t}=-\int_{\mathcal{V}} \boldsymbol{r} \times \rho \frac{\partial \boldsymbol{\omega}}{\partial t} \mathrm{~d} \mathcal{V} \\
\boldsymbol{F}_{S_{f a r}}=-\int_{S_{f a r}} \boldsymbol{r} \times(\boldsymbol{n} \times \rho \boldsymbol{\ell}) \mathrm{d} S, & \boldsymbol{F}_{S_{b}}=-\int_{S_{b}} \boldsymbol{r} \times(\boldsymbol{n} \times \rho \boldsymbol{\ell}) \mathrm{d} S, \\
\boldsymbol{F}_{t_{B}}=\int_{S_{b}} \boldsymbol{r} \times(\boldsymbol{n} \times \rho \boldsymbol{a}) \mathrm{d} S, & \boldsymbol{\Delta}_{\boldsymbol{\mu}}=\int_{S_{f a r}} \boldsymbol{r} \times \boldsymbol{n} \times \boldsymbol{\nabla} \cdot \boldsymbol{\tau}_{v} \mathrm{~d} S+\int_{S_{f a r}} \boldsymbol{\tau}_{v} \cdot \boldsymbol{n} \mathrm{d} S,
\end{aligned}
$$

with $\boldsymbol{r}$ the position vector with respect to an arbitrary pole. $\boldsymbol{F}_{t}$ and $\boldsymbol{F}_{S_{b}}$ are related with $I=\rho \int_{\mathcal{V}} \boldsymbol{r} \times \boldsymbol{\omega} \mathrm{d} \mathcal{V}$, the hydrodynamic impulse in $\mathcal{V}$. Indeed, taking into account for Reynolds transport theorem with $S_{\text {far }}$ fixed in time, we have:

$$
\boldsymbol{F}_{t}+\boldsymbol{F}_{S_{b}}=-\frac{d I}{d t} \equiv \boldsymbol{F}_{\tau} .
$$

Equation (9) also holds in three-dimensional flow, provided $\boldsymbol{r}$ is replaced by $\tilde{\boldsymbol{r}}=\boldsymbol{r} / 2$.

\section{B. Compressible regime}

Mele and Tognaccini [16] derived an exact expression of aerodynamic force in compressible flows, again in terms of the vortex force, that is

$$
\boldsymbol{F}_{b}=\boldsymbol{F}_{\ell}+\boldsymbol{F}_{m_{\rho}}+\boldsymbol{F}_{t \rho}+\boldsymbol{F}_{S_{f a r}}+\boldsymbol{F}_{S_{b}}+\boldsymbol{F}_{t_{b}}+\Delta_{\mu}
$$

where

$$
\boldsymbol{F}_{m_{\rho}}=-\int_{\mathcal{V}} \boldsymbol{r} \times \boldsymbol{\nabla} \times \rho \boldsymbol{\nabla}\left(\frac{V^{2}}{2}\right) \mathrm{d} \mathcal{V}=-\int_{\mathcal{V}} \boldsymbol{m}_{\rho} \mathrm{d} \mathcal{V}, \quad \boldsymbol{F}_{t \rho}=-\int_{\mathcal{V}} \boldsymbol{r} \times \boldsymbol{\nabla} \times \rho \frac{\partial \boldsymbol{V}}{\partial t} \mathrm{~d} \mathcal{V} .
$$

$\boldsymbol{F}_{m_{\rho}}$ is an additional compressibility correction for the vortex force, while $\boldsymbol{F}_{t \rho}$ is an unsteady term that, differently from $\boldsymbol{F}_{t}$ takes also into account of compressibility effects. $\boldsymbol{\Delta}_{\mu}$ is already negligible for moderately high Reynolds number flows. In steady flows a force breakdown by means of this formula has been introduced [17]. Specifying with $\boldsymbol{i}_{x}$ and $\boldsymbol{i}_{z}$ the unit versors aligned and orthogonal to the freestream, we have:

$$
\begin{aligned}
D_{p} & =-\boldsymbol{i}_{x} \cdot \int_{S_{\text {far }}} \tilde{\boldsymbol{r}} \times(\boldsymbol{n} \times \rho \boldsymbol{\ell}) \mathrm{d} \mathrm{S}, \\
L & =-\boldsymbol{i}_{z} \cdot \int_{\mathcal{V}}\left(\rho \boldsymbol{\ell}+\boldsymbol{m}_{\rho}\right) \mathrm{d} \boldsymbol{V}, \\
D_{i} & =-\boldsymbol{i}_{x} \cdot \int_{\mathcal{V}}\left(\rho \boldsymbol{\ell}+\boldsymbol{m}_{\rho}\right) \mathrm{d} \boldsymbol{V} .
\end{aligned}
$$

where $D_{p}$ is the profile drag (including wave contribution), $L$ is the aerodynamic lift and $D_{i}$ is the lift-induced drag. 


\section{Link with inviscid theories}

\section{Incompressible regime}

Very recently, Ostieri et al. [19], [20] found the link between present aerodynamic force theory and inviscid results. Considering a two dimensional incompressible inviscid flow around a flat plate, of length $2 b$, centered at $x=0$, and integrating the pressure loading $\Delta p$ from $-b$ to $x_{w}$, where $x_{w}>b$ is a wake station, and taking into account that $\Delta p$ is 0 on the wake:

$$
l=-\int_{-b}^{x_{w}} \Delta p(x, t) \mathrm{d} x=\rho V_{\infty} \int_{-b}^{x_{w}} \gamma(\xi, t) \mathrm{d} x+\rho \frac{d}{d t} \int_{-b}^{x_{w}}\left(x_{w}-\xi\right) \gamma(\xi, t) \mathrm{d} \xi
$$

where $\gamma$ is the concentrated vorticity distribution over the flat plate, and on the wake. Similarly, for a viscous flow, taking into account for identity (11), valid for incompressible flows, equation (9) gives:

$$
\boldsymbol{F}=-\rho \int_{\mathcal{V}} \boldsymbol{\ell} \mathrm{d} \mathcal{V}-\frac{\mathrm{d}}{\mathrm{d} t} \int_{\mathcal{V}} \boldsymbol{r} \times \boldsymbol{\omega} \mathrm{d} \mathcal{V}-\rho \int_{S_{f a r}} \boldsymbol{r} \times \boldsymbol{n} \times \boldsymbol{\ell} \mathrm{d} S .
$$

This formula is valid for an arbitrary choice of the pole. Considering $S_{f a r}$ composed by a wake plane $S_{w}$ orthogonal to the wake and adopting as pole for the moment calculations the wake center on $S_{w}\left(\boldsymbol{r}_{w}\right)$, we have

$$
\boldsymbol{F}=-\rho \int_{\mathcal{V}} \boldsymbol{\ell} \mathrm{d} \mathcal{V}-\frac{\mathrm{d}}{\mathrm{d} t} \int_{\mathcal{V}}\left(\boldsymbol{r}-\boldsymbol{r}_{w}\right) \times \omega \mathrm{d} \mathcal{V}-\rho \int_{S_{f a r}}\left(\boldsymbol{r}-\boldsymbol{r}_{w}\right) \times \boldsymbol{n} \times \boldsymbol{\ell} \mathrm{d} S .
$$

The connection of the first two terms of equation (18) with the inviscid formula (16) is evident. The last term of equation [18) is only a drag contribution [16]. Furthermore, integration by part of this term gives the momentum defect on the wake [21], therefore it takes into account for viscous effects, and is the irreversible contribution to the aerodynamic force:

$$
\boldsymbol{F}_{\text {irr }}=-\rho \int_{S_{\text {far }}}\left(\boldsymbol{r}-\boldsymbol{r}_{w}\right) \times \boldsymbol{n} \times \boldsymbol{\ell} \mathrm{d} S .
$$

The reversible part of the aerodynamic force is given by the remaining part, responsible for the lift generation:

$$
\begin{aligned}
\boldsymbol{F}_{r e v} & =-\rho \int_{\mathcal{V}} \boldsymbol{\ell} \mathrm{d} \mathcal{V}+\rho \frac{\mathrm{d}}{\mathrm{d} t} \int_{\mathcal{V}}\left(\boldsymbol{r}_{w}-\boldsymbol{r}\right) \times \omega \mathrm{d} \mathcal{V}= \\
& =-\rho \int_{\mathcal{V}} \boldsymbol{\ell} \mathrm{d} \mathcal{V}+\rho \int_{\mathcal{V}}\left(\boldsymbol{r}_{w}-\boldsymbol{r}\right) \times \frac{\partial \boldsymbol{\omega}}{\partial t} \mathrm{~d} \mathcal{V}+\rho \int_{S_{b}}\left(\boldsymbol{r}_{w}-\boldsymbol{r}\right) \times \boldsymbol{n} \times \boldsymbol{\ell} \mathrm{d} S
\end{aligned}
$$

Equation 20 is the perfect counterpart of equation (16) in viscous flows. With some algebra, we can obtain another expression of $\boldsymbol{F}_{\text {irr }}$ :

$$
\boldsymbol{F}_{i r r}=-\rho \int_{S_{f a r}} \boldsymbol{r} \times \boldsymbol{n} \times \boldsymbol{\ell} \mathrm{d} S-\rho \int_{\mathcal{V}} \boldsymbol{r}_{w} \times \frac{\partial \boldsymbol{\omega}}{\partial t} \mathrm{~d} \mathcal{V}+\rho \int_{S_{b}} \boldsymbol{r}_{w} \times \boldsymbol{n} \times \boldsymbol{\ell} \mathrm{d} S
$$

The definition of $\boldsymbol{F}_{r e v}$ and $\boldsymbol{F}_{i r r}$ is rigorous as far as the definition of $\boldsymbol{r}_{w}$ is clear. Indeed, while $x_{w}$ in an inviscid flow can be chosen arbitrarily, in a real viscous flow $x_{w}$ has to be placed at the end of the near wake, where for near wake here we intend the flow zone just after the body TE where the effect of the body vorticity (bound vorticity) is still relevant.

As opposed to the thermodynamic method, the Lamb vector formula can also compute and decompose lift.

\section{An extension to compressible flows}

An extension of the definition of reversible and irreversible force components in the compressible regime is here proposed. Proposed formulae are consistent with the incompressible breakdown. We have:

$$
\begin{gathered}
\boldsymbol{F}_{r e v}=\boldsymbol{F}_{\ell}+\boldsymbol{F}_{m_{\rho}}+\boldsymbol{F}_{t *} \\
\boldsymbol{F}_{\text {irr }}=-\int_{S_{\text {far }}} \boldsymbol{r} \times \boldsymbol{n} \times \rho \boldsymbol{\ell} \mathrm{d} S+\int_{\mathcal{V}} \boldsymbol{r}_{w} \times \frac{\partial \rho \boldsymbol{\omega}}{\partial t} \mathrm{~d} \boldsymbol{V}+\int_{S_{b}} \boldsymbol{r}_{w} \times \boldsymbol{n} \times \rho \boldsymbol{\ell} \mathrm{d} S
\end{gathered}
$$

where $\boldsymbol{F}_{t *}$ :

$$
\boldsymbol{F}_{t *}=-\int_{\mathcal{V}} \boldsymbol{r} \times \boldsymbol{\nabla} \times \rho \frac{\partial \boldsymbol{V}}{\partial t} \mathrm{~d} \mathcal{V}+\int_{\mathcal{V}} \boldsymbol{r}_{w} \times \frac{\partial \rho \boldsymbol{\omega}}{\partial t} \mathrm{~d} \mathcal{V}+\int_{S_{b}}\left(\boldsymbol{r}_{w}-\boldsymbol{r}\right) \times \boldsymbol{n} \times \rho \boldsymbol{\ell} \mathrm{d} S
$$




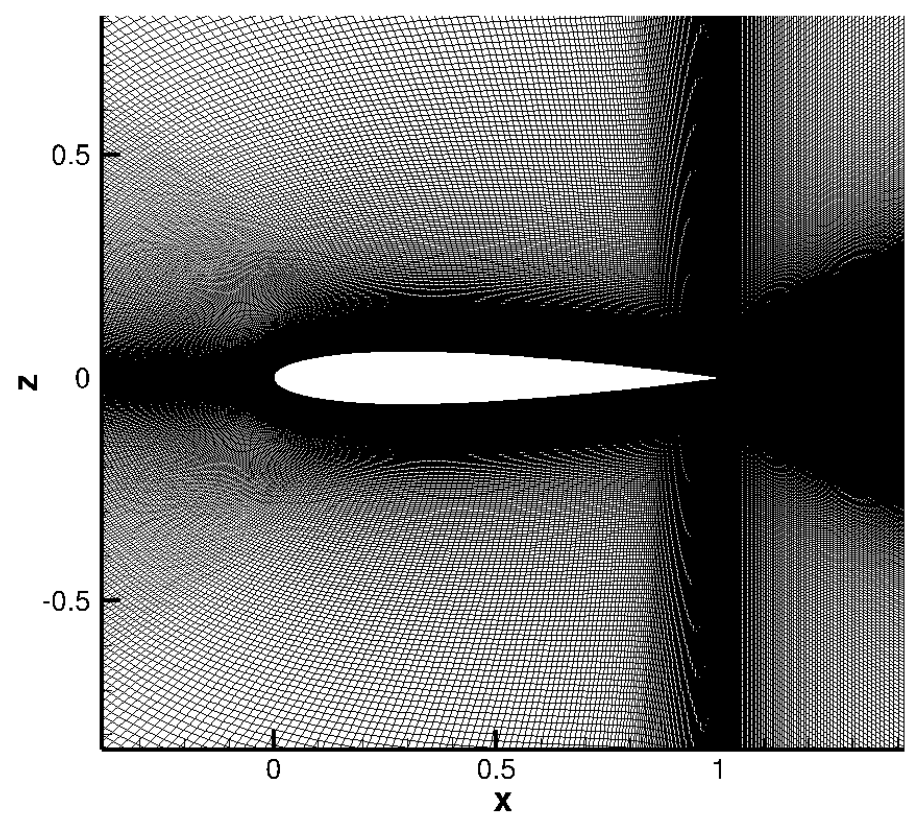

Fig. 1 Detail of the computational mesh used for the pitching airfoil test case.

\section{Numerical applications}

\section{A. Pitching airfoil}

The numerical solution around a pitching NACA 0012 here analyzed is the same performed by Toubin and Bailly [8]. Simulations were performed with ONERA elsA code [22]. The imposed pitching motion is sinusoidal, with an oscillation between $-5^{\circ}$ and $+5^{\circ}$. The freestream Mach number is $M_{\infty}=0.3$, the Reynolds number $R e_{\infty}=6.6 \cdot 10^{6}$, the reduced frequency $k=0.1$. 9000 time steps for each time period have been set. Spalart-Allmaras turbulence model and Jameson numerical scheme have been adopted. The grid is composed of about 530000 cells, with 568 body points. Grid near the body is shown in figure 1 . The farfield is placed at 20 chords distance in the downstream direction, and 25 chords in the others. Vorticity contours at different timesteps are shown in figure 2

In figure 3 computed lift coefficient has been analyzed. Equation (12) and near field computation are in good agreement. The same result is also obtained by $C_{l_{\text {rev }}}$. For this test case a weak sensitivity to the choice of the integration domain was found for the lift coefficient. In figure 4 the computed drag coefficients are proposed as obtained by eqs. (1) and (12). Both formulae are in very good agreement with the near field computation. Again, as for the lift coefficient, total drag is not sensitive to the chosen integration domain.

Drag breakdown To obtain the drag breakdown in reversible and irreversible contributions by Lamb vector method, a right choice of $x_{w}$ is required. Indeed, the strongest limitation of the vortical method is that drag breakdown changes significantly with the value of $x_{w}$. Here a strategy is proposed for a correct $x_{w}$ definition. The vortex drag, as obtained by varying the integration domain is displayed at two different time steps in figure 5 . The abscissa $x_{w}$ represents the position of the wake plain chosen for the integration. In this test, vortex drag, directly proportional to the local vorticity, has a sudden increase just after the TE, then shows a periodical-like behavior, similar to the periodical wake solution predicted in inviscid incompressible flows. This behavior starts at $x_{w} \approx 0.4$. For this test this $x_{w}$ had only very small variations with time. All results proposed have been obtained with $x_{w}=0.4$.

The two drag breakdowns are compared in figure 6 . The decomposition performed by equation (1) was already presented by Toubin and Bailly [8]. $C_{d_{v}}$ and $C_{d_{i r} r}$ values are similar: differences are within 10 drag counts. In particular, the agreement between $C_{d_{u i}}$ and $C_{d_{r e v}}$ is more satisfactory. Please note that, while the results of the thermodynamic 


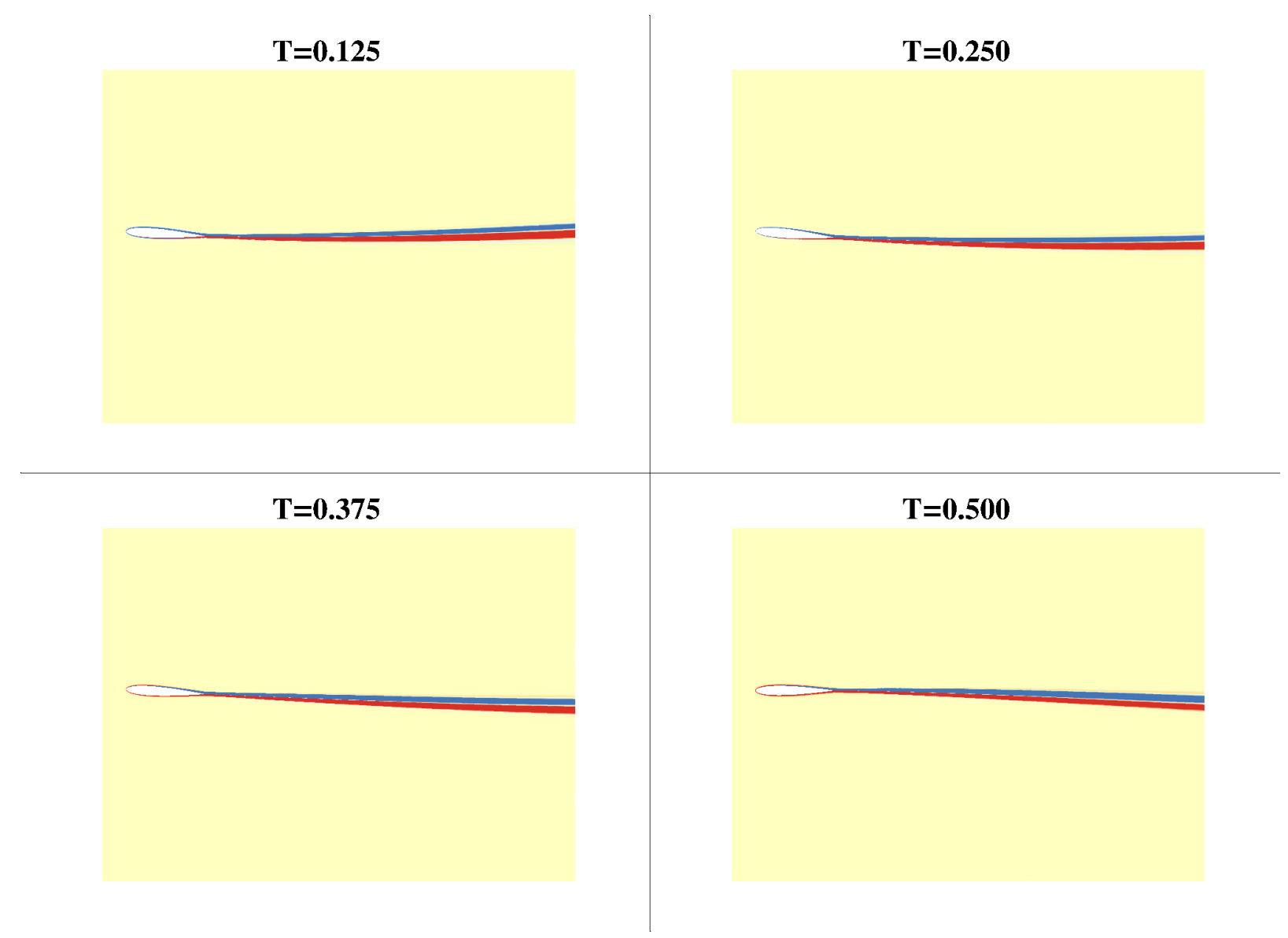

Fig. 2 Pitching NACA 0012, $R e_{\infty}=6.6 \cdot 10^{6}, M_{\infty}=0.3, \bar{\alpha}=5^{\circ}, k=0.1$. Vorticity contours at different timesteps.

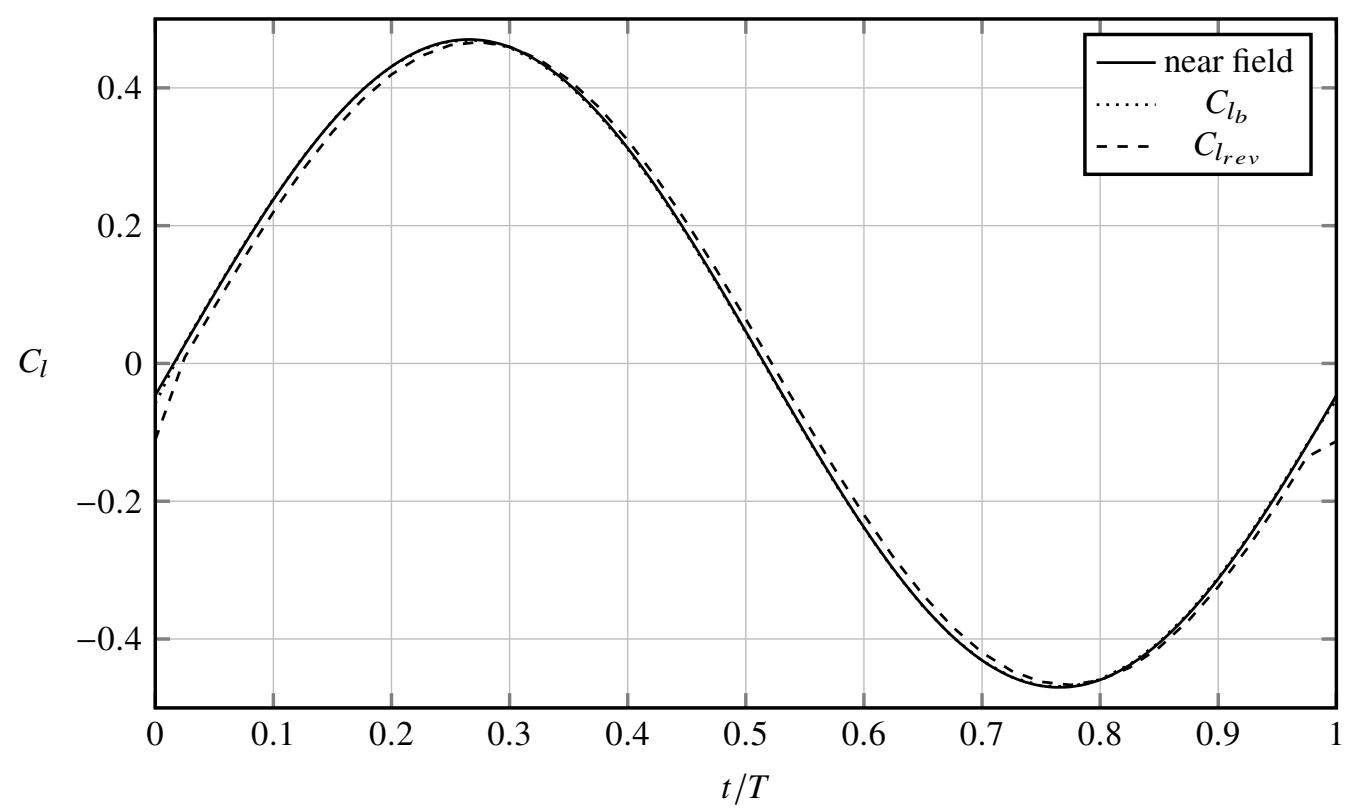

Fig. 3 Pitching NACA 0012, $R e_{\infty}=6.6 \cdot 10^{6}, M_{\infty}=0.3, \bar{\alpha}=5^{\circ}, k=0.1$. Computed aerodynamic lift coefficients. 


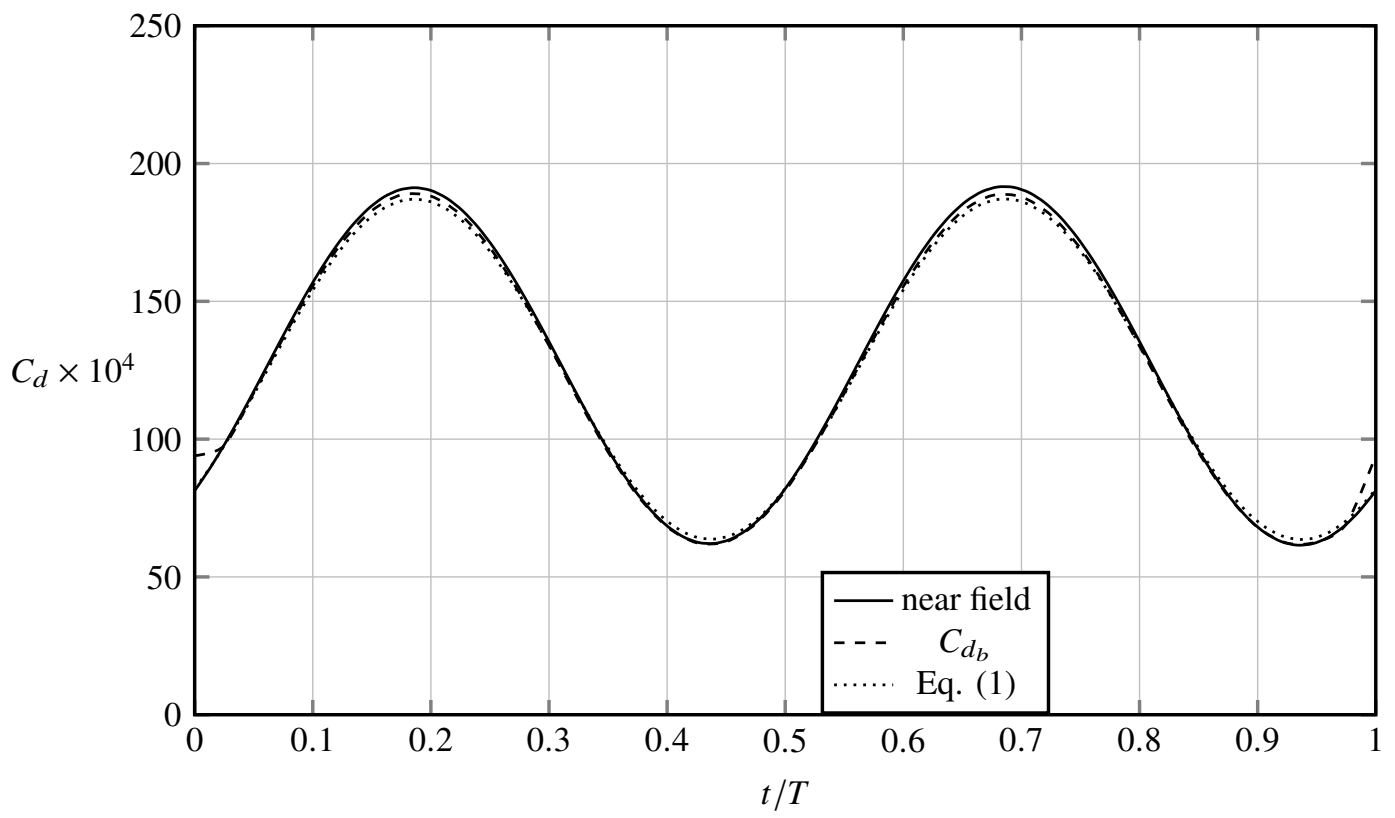

Fig. 4 Pitching NACA 0012, $R e_{\infty}=6 \cdot 10^{6}, M_{\infty}=0.3, \bar{\alpha}=5^{\circ}, k=0.1$. Computed aerodynamic drag coefficient.

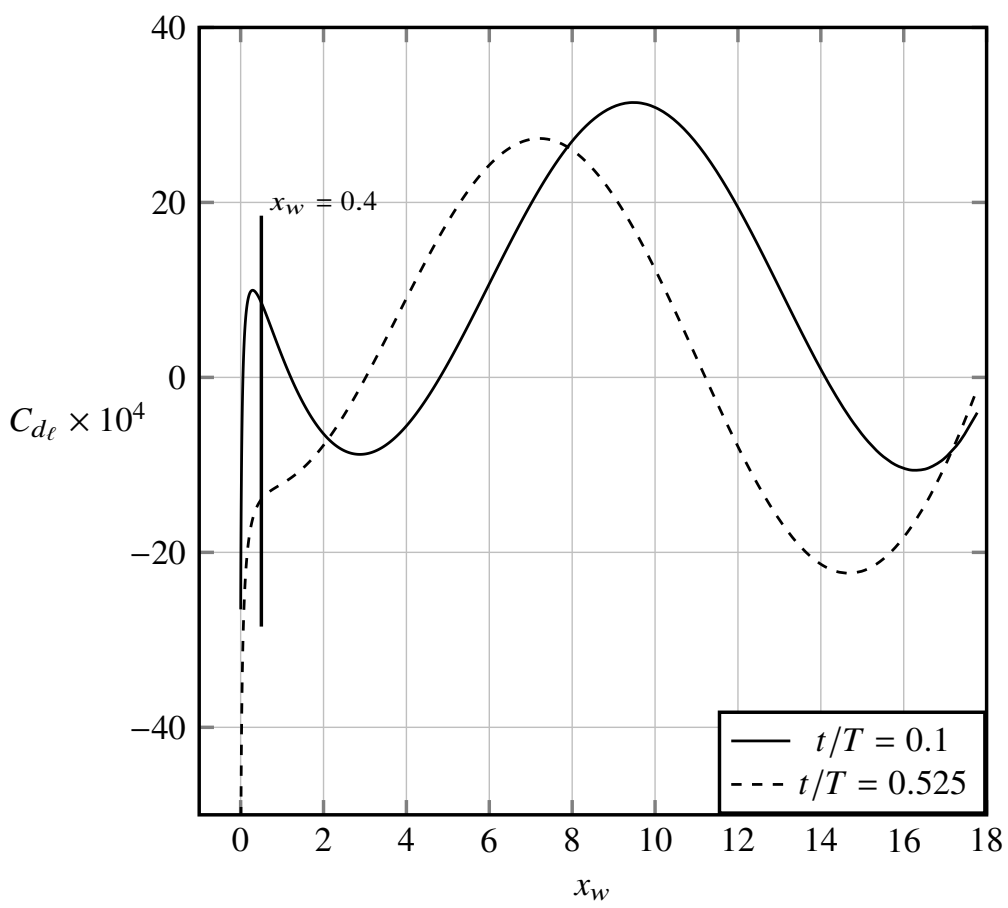

Fig. 5 Pithing NACA 0012, $R e_{\infty}=6.6 \cdot 10^{6}, M_{\infty}=0.3, \bar{\alpha}=5^{\circ}, k=0.1$. Vortex drag distribution from the TE to $x_{w}$ at different timesteps. 


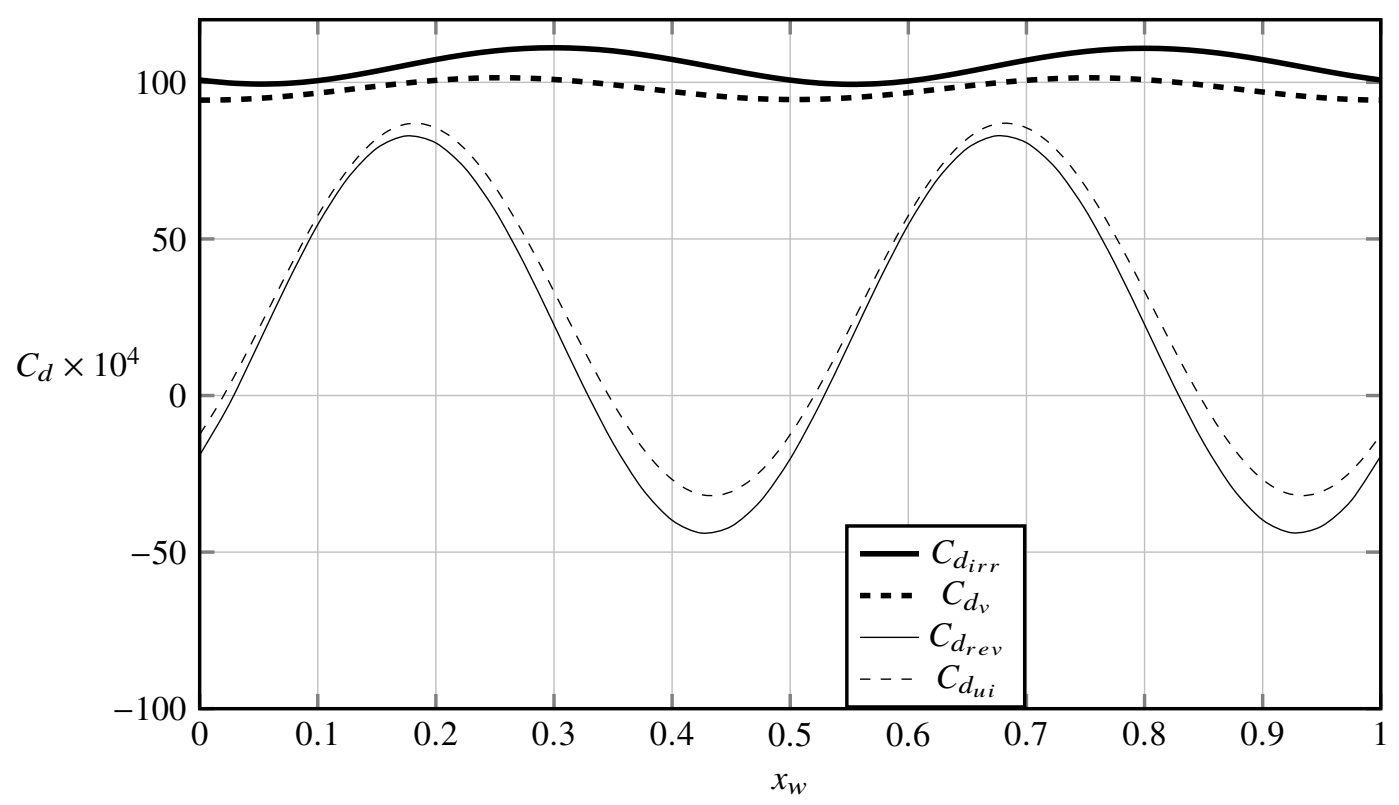

Fig. 6 Pitching NACA 0012, $R e_{\infty}=6 \cdot 10^{6}, M_{\infty}=0.3, t / T=0.125, k=0.1$. Comparison between proposed drag components.

method are weakly sensitive to the chosen integration domain, the proposed vorticity based reversible and irreversible breakdown is much more dependent on the choice of the wake plane.

\section{B. Airfoil buffet}

The unsteady RANS simulation of the buffet around an OAT15A airfoil is the same reported in [8]. Reynolds number is $R e_{\infty}=3 \cdot 10^{6}$; freestream Mach number is $M_{\infty}=0.73$, and angle of attack $\alpha=4.5^{\circ} . \kappa-\omega$ Wilcox turbulence model and Roe numerical scheme have been used. The grid domain extends about 40 airfoil chords downstream, upstream and laterally. Mesh is built up of about 150000 cells, with 679 body points. A detail of the grid near the body is shown in figure 7. Each oscillation period is composed of 1000 time steps, with a frequency of $60 \mathrm{~Hz} .5$ sub-iterations only were adopted in the dual time stepping procedure. Vorticity contours at different timesteps are shown in figure 8

Numerical issues affected the analysis of the aerodynamic force by equation (12). Indeed, differently from equation (1), vortical method requires the computation of space derivatives and volume integrals in the zones where accuracy is poorer, such as the shock region and the wake, where the grid is coarser. The dual-time stepping used was good enough to obtain a converged near-field force. On the contrary, the analysis with the vortical method requires a deeper convergence; indeed sufficient agreement with the near field prediction was only possible choosing the boundary of the integration domain very near the airfoil as shown in figures 9 and 10 . In this figures, $x_{s}$ and $y_{s}$ specify the size of the integration domain, $x_{s}$ is the position of the wake plane, whereas $y_{s}$ the lateral distance.

Drag breakdown The same strategy applied to the pitching airfoil test case is here proposed. Vortex drag variation while changing wake plane position at different timesteps is shown in figure 11. In this case was not possible to find a value for $x_{w}$. The analysis was carried out varying $x_{w}$ and comparing the vortical breakdown with the thermodynamic one.

In figure 12 is shown the breakdown proposed by Toubin and Bailly [8]. The decomposition between viscous and wave components is proposed. The proposed induced drag has a positive small mean value, while $D_{p a}$ is larger. Wave drag varies significantly, following the behavior of the shock during the buffet [8]. The drag breakdown by vortical method is proposed in figure with a comparison with the thermodynamic decomposition. The value choosen for $x_{w}$ is 4 . There is a qualitative agreement between proposed breakdown in magnitude, but not in phase with the time. At higher values of $x_{w}$ the phase tends to become the same of the thermodynamic breakdown, but the magnitude is affected by the numerical errors which affects the total drag computation. 


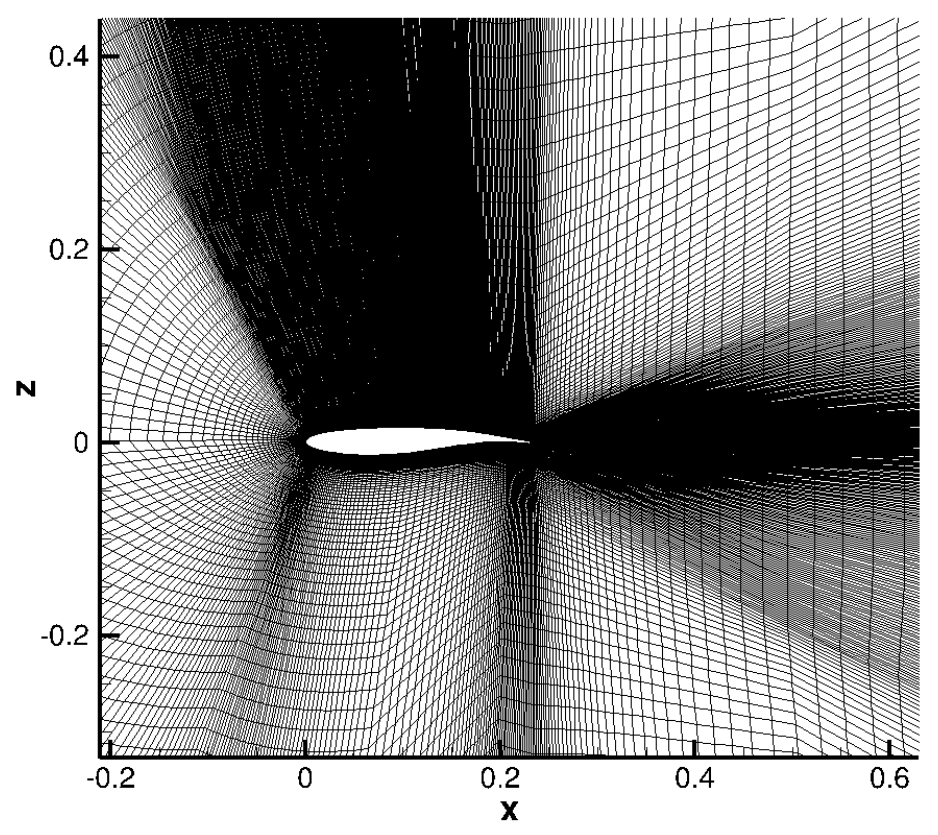

Fig. 7 Detail of the computational mesh used for the airfoil buffet test case.

\section{Conclusions}

Two different aerodynamic force breakdown methods, valid in unsteady and compressible viscous flow, have been here compared. The first technique, proposed by Toubin and Bailly [8] is based on a local irreversible velocity definition that relies on local entropy production, here referred as thermodynamic method. This method cannot compute aerodynamic lift, but can accurately compute aerodynamic drag as shown by Toubin and Bailly [8], Toubin et al. [9]. The second method is based on a Lamb-vector based theory [19, 20], here referred as vortical method. This method, that can compute not only aerodynamic drag, but also the aerodynamic lift, permitted for a new definition of reversible and irreversible contributions to the aerodynamic force, where the first takes into account for local entropy production effects, and the second is connected to the lift generation.

Numerical applications have been proposed for two physically completely different test-cases: a pitching NACA 0012 in subsonic flow and an airfoil natural buffet. Toubin and Bailly drag breakdown results have been compared with the proposed vortical force breakdown. As concerning the pitching airfoil test case, total aerodynamic lift is correctly computed by the vortical method, and the reversible contribution to the force correctly computes all the aerodynamic lift. Total aerodynamic drag computed by both methods is in perfect agreement with near field computations. The numerical results revealed a satisfactory quantitative agreement between proposed drag decompositions. In the second test case, total force computed by the vortical method is again in satisfactory agreement with the near field results. The comparison between thermodynamic and vortical breakdown revealed not sufficiently satisfactory, with a qualitative agreement in magnitude but not in phase. Further improvements are necessary for the vortical decomposition, in order to obtain a breakdown formulation not dependent on the integration domain.

\section{References}

[1] Betz, A., "A method for the direct determination of wing-section drag," Tech. Rep. 337, NACA Technical Report, 1925.

[2] Oswatitsch, K., Gas Dynamics, Academic Press, New York, US, 1956. 
$\mathbf{T}=\mathbf{0 . 2 5}$

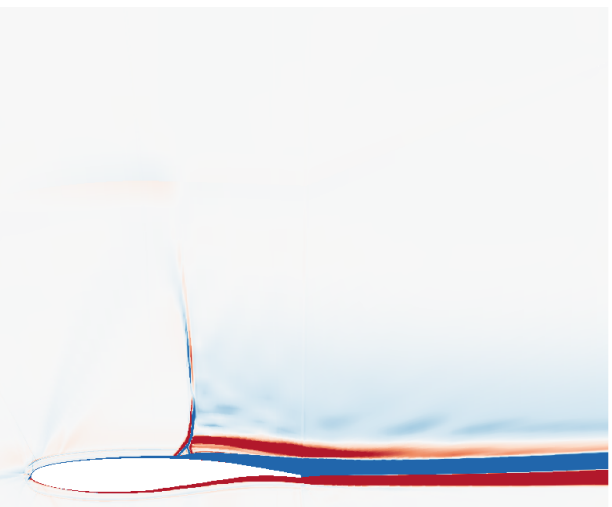

$\mathbf{T}=\mathbf{0 . 7 5}$

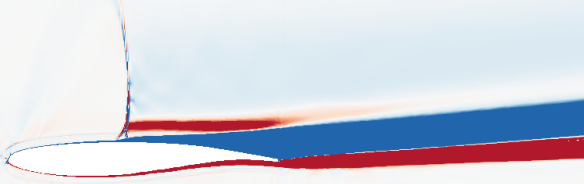

Fig. 8 Airfoil OAT15A, $R e_{\infty}=3 \cdot 10^{6}, M_{\infty}=0.73, \bar{\alpha}=4.5^{\circ}$. Vorticity contours at different timesteps.

$x_{w} / c=2$

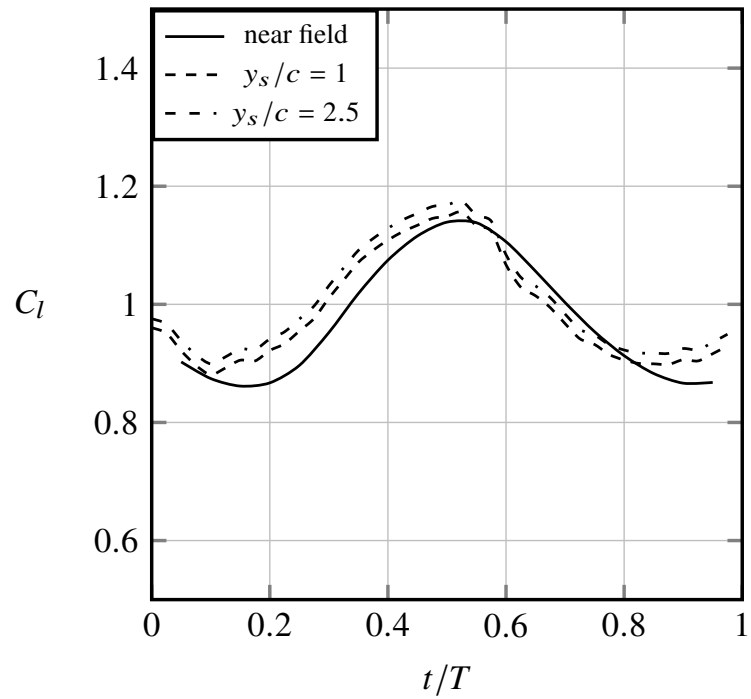

$y_{s} / c=1$

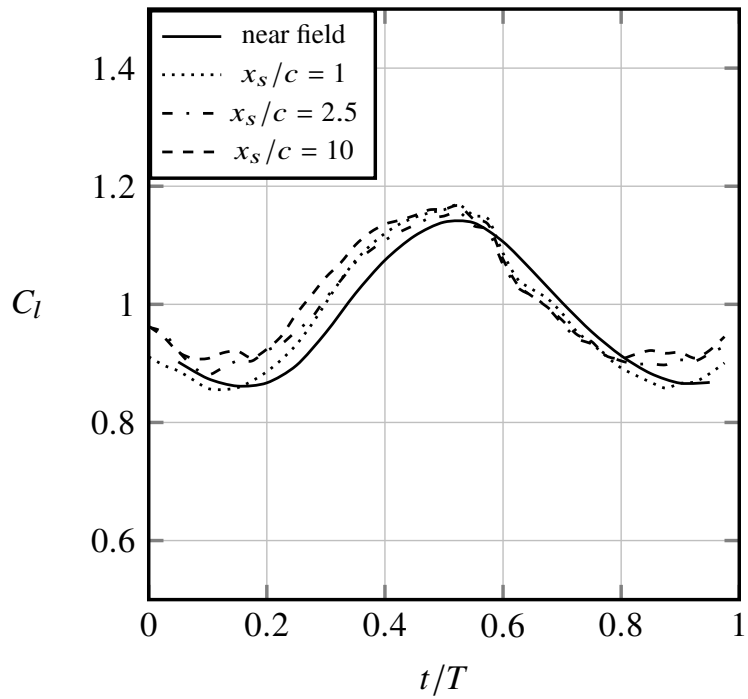

Fig. 9 Buffet test case, Airfoil OAT15A, $R e_{\infty}=3 \cdot 10^{6}, M_{\infty}=0.73, \bar{\alpha}=4.5^{\circ}$. Near field vs midfield drag computations. 
$x_{s} / c=2$

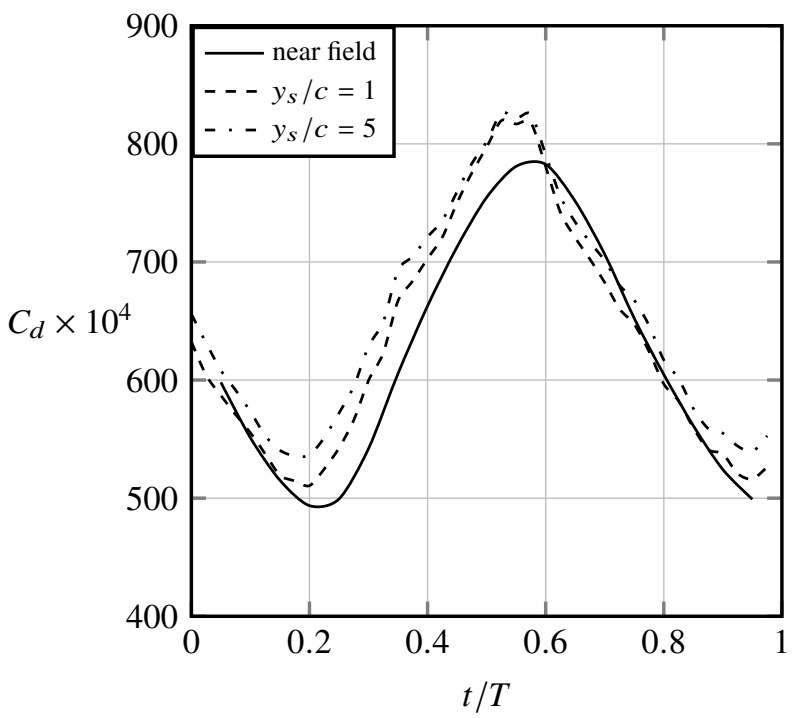

$y_{s} / c=1$

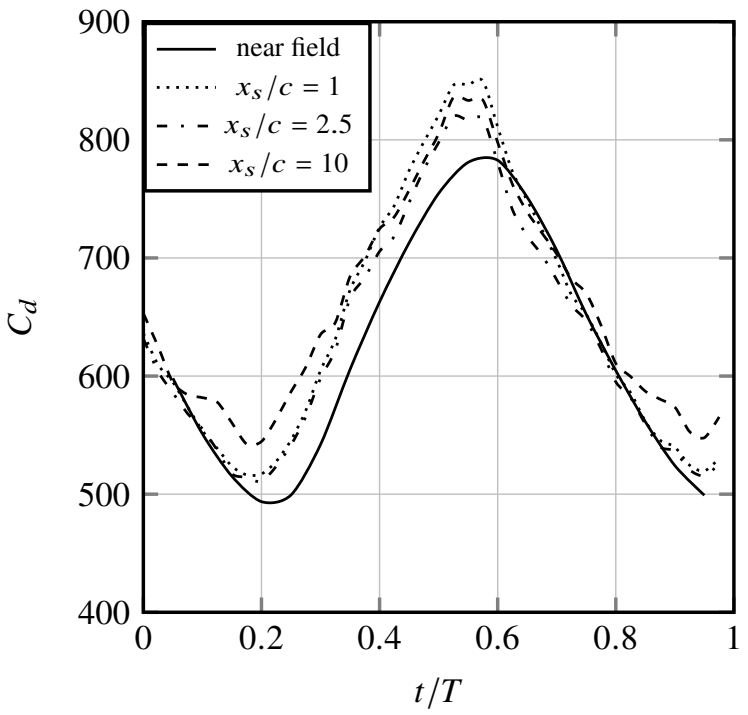

Fig. 10 Buffet test case, Airfoil OAT15A, $R e_{\infty}=3 \cdot 10^{6}, M_{\infty}=0.73, \bar{\alpha}=4.5^{\circ}$. Near field vs midfield drag computations.

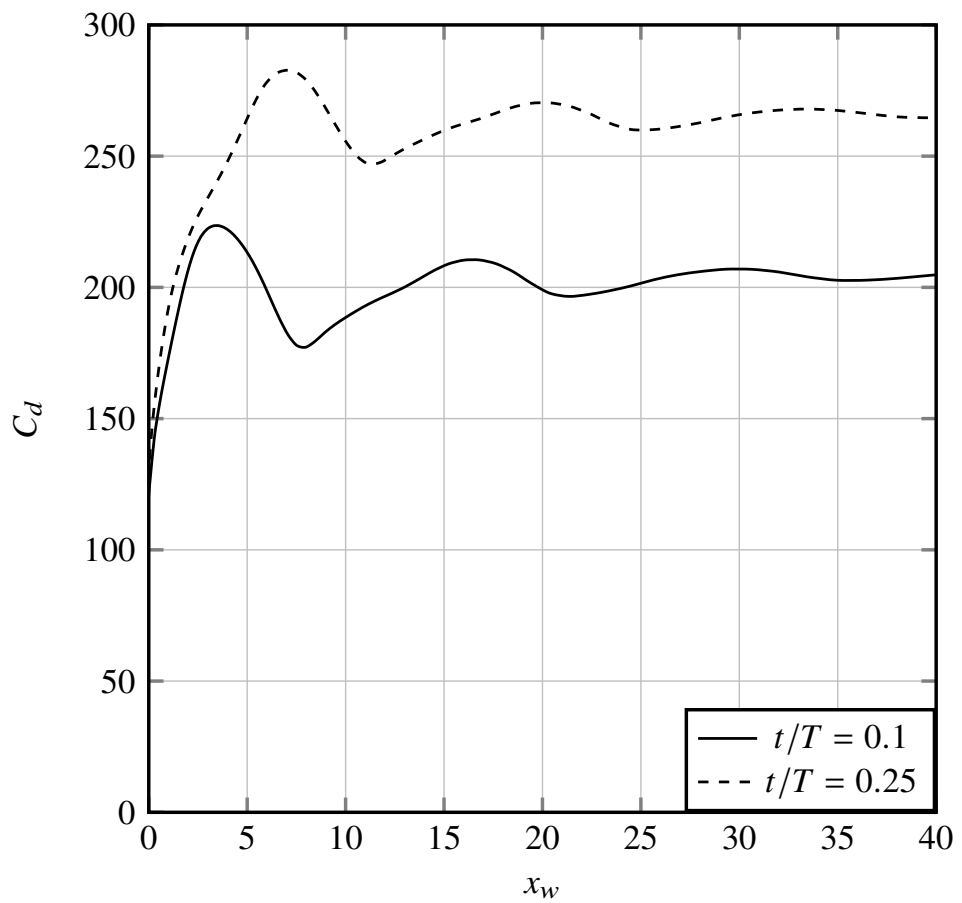

Fig. 11 Buffet test case, airfoil OAT15A, $R e_{\infty}=3 \cdot 10^{6}, M_{\infty}=0.73, t / T=0.1$. Vortex drag distribution from the TE to $x_{w}$. 


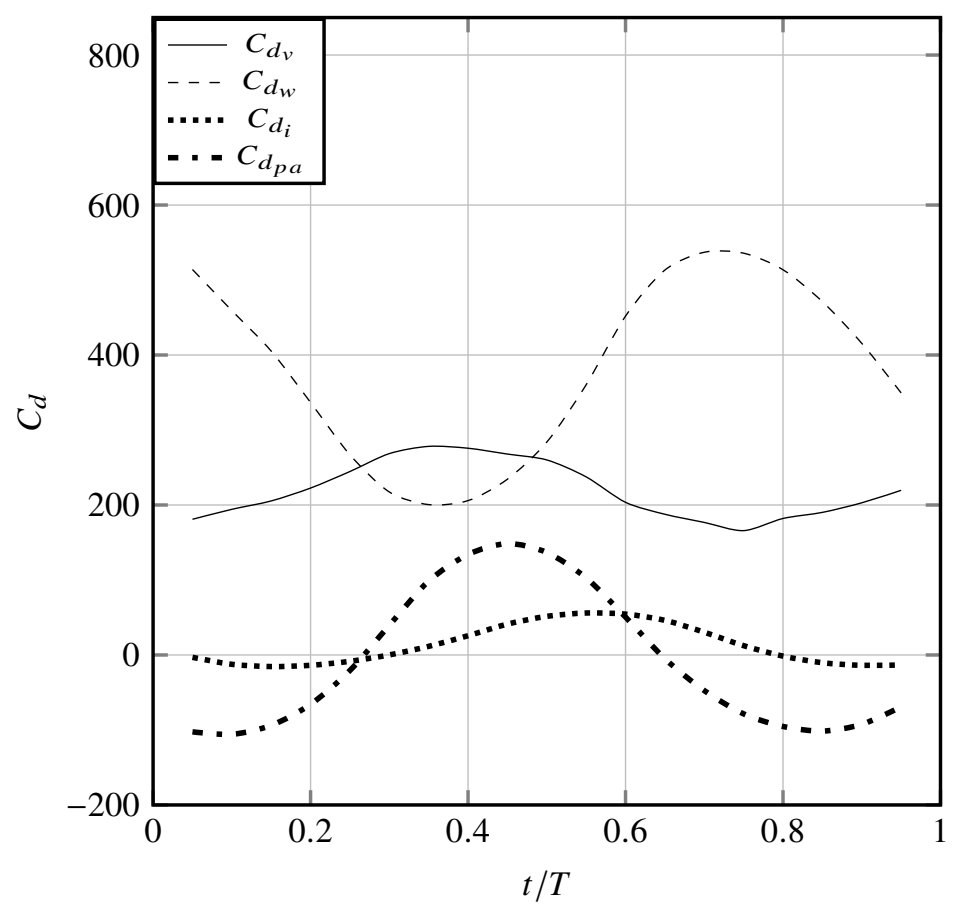

Fig. 12 Buffet test case, airfoil OAT15A, $R e_{\infty}=3 \cdot 10^{6}, M_{\infty}=0.73, \bar{\alpha}=4.5^{\circ} \cdot x_{s}=2, y_{s}=1$. Proposed drag breakdown by Toubin and Bailly [8]

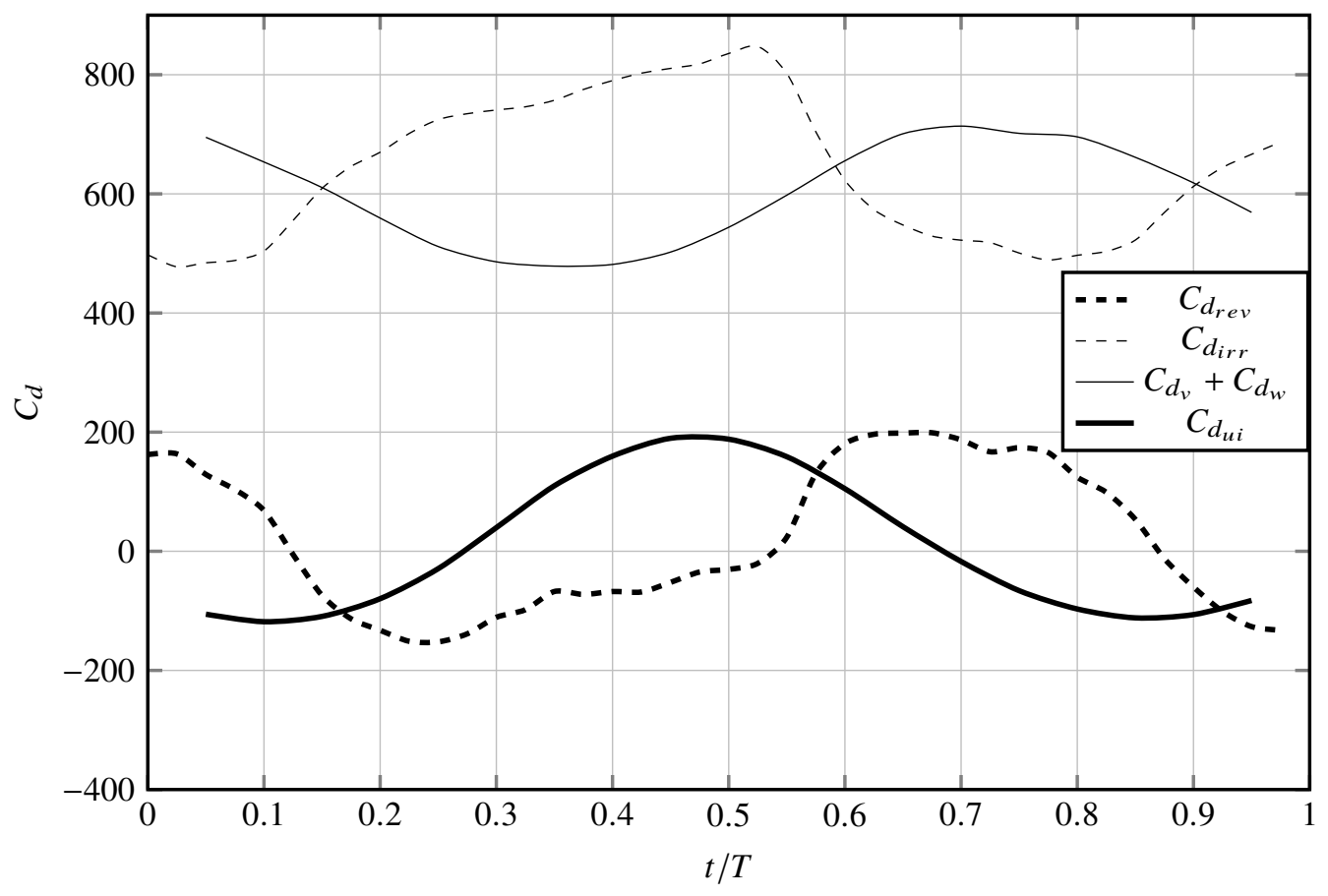

Fig. 13 Buffet test case, airfoil OAT15A, $R e_{\infty}=3 \cdot 10^{6}, M_{\infty}=0.73, \bar{\alpha}=4.5^{\circ} . x_{s}=2, y_{s}=1$. Drag breakdown comparison, $x_{w}=0.4$. 
[3] Maskell, E., "Progress towards a method for the measurement of the components of the drag of a wing of finite span,” Tech. rep., RAE Technical Report No. 72232, United Kingdom, 1972.

[4] Paparone, L., and Tognaccini, R., "Computational Fluid Dynamics-based drag prediction and decomposition," AIAA Journal, Vol. 41, No. 9, 2003, pp. 1647-1657. doi:10.2514/2.7300.

[5] van der Vooren, J., and Destarac, D., "Drag/thrust analysis of jet-propelled transonic transport aircraft; definition of physical drag components," Aerospace Science and Technology, Vol. 8, 2004, pp. 545-556.

[6] Tognaccini, R., "Drag computation and breakdown in power-on conditions," Journal of Aircraft, Vol. 42, No. 1, 2005, pp. 245-252. doi:10.2514/1.8510.

[7] Gariepy, M., Trepanier, J. Y., and Malouin, B., "Generalization of the far-field drag decomposition method to unsteady flows," AIAA Journal, Vol. 51, No. 6, 2013, pp. 1309-1319.

[8] Toubin, H., and Bailly, D., "Development and application of a new unsteady far-field drag decomposition method," AIAA Journal, Vol. 52, No. 11, 2015, pp. 3414-3429.

[9] Toubin, H., Bailly, D., and Costes, M., "Improved Unsteady Far-Field Drag Breakdown Method and Application to Complex Cases," AIAA Journal, Vol. 54, No. 6, 2016, pp. 1907-1921.

[10] Wu, J., Lu, X.-Y., and Zhuang, L.-X., "Integral force acting on a body due to local flow structures," Journal of Fluid Mechanics, Vol. 576, 2007, pp. 265-286.

[11] Marongiu, C., and Tognaccini, R., "Far-field analysis of the Aerodynamic force by Lamb vector integrals," AIAA Journal, Vol. 48, No. 11, 2010, pp. 2543-2555. doi:10.2514/1.J050326.

[12] Marongiu, C., Tognaccini, R., and Ueno, M., "Lift and lift-induced drag calculation by Lamb vector integration," AIAA Journal, Vol. 51, No. 6, 2013, pp. 1420-1430. doi:10.2514/1.J052104.

[13] Wu, J., Ma, H.-Y., and Zhou, M.-D., Vorticity and Vortex Dynamics, Springer, 2006.

[14] Liu, L., Wu, J., Shi, Y., and Zhu, J., "A dynamic counterpart of Lamb vector in viscous compressible aerodynamics," Fluid Dynamics Research, Vol. 46, 2014, pp. 1-14.

[15] Liu, L., Shi, Y., Zhu, J., Su, W., Zou, S., and Wu, J., "Longitudinal-transverse aerodynamic force in viscous compressible complex flow," Journal of Fluid Mechanics, Vol. 756, 2014, pp. 226-251.

[16] Mele, B., and Tognaccini, R., "Aerodynamic force by Lamb vector integrals in compressible flow," Physics of Fluids, Vol. 26, 2014, pp. 056104-1-16. doi:10.1063/1.4875015.

[17] Mele, B., Ostieri, M., and Tognaccini, R., "Vorticity based breakdown of the aerodynamic force in three-dimensional compressible flows," AIAA Journal, Vol. 54, No. 4, 2016, pp. 1198-1208.

[18] Mele, B., Ostieri, M., and Tognaccini, R., "Aircraft lift and drag decomposition in transonic flows," Journal of Aircraft, Vol. 54, No. 5, 2017, pp. 1933-1944.

[19] Ostieri, M., Mele, B., and Tognaccini, R., "Exact aerodynamic force decomposition and dynamic force derivatives in non linear flows," AIAA SciTech Forum - 55th AIAA Aerospace Sciences Meeting, 2017.

[20] Ostieri, M., Mele, B., and Tognaccini, R., "Linear and nonlinear decomposition of the aerodynamic force acting on an oscillating plate," AIAA Journal, 2017. Article in advance.

[21] Wu, J. C., "Theory for Aerodynamic force and moment in viscous flows," AIAA Journal, Vol. 19, No. 4, 1981, pp. $432-441$.

[22] Cambier, L., and Gazaix, M., "ElsA: An efficient object-oriented solution to CFD complexity," 40th AIAA Aerospace Sciences Meeting and Exhibit, 2002. 